Pacific Journal of Mathematics

A LOOP SPACE WHOSE HOMOLOGY HAS TORSION OF ALL

David JY ANIC 


\title{
A LOOP SPACE WHOSE HOMOLOGY HAS TORSION OF ALL ORDERS
}

\author{
DAVID J. ANICK
}

\begin{abstract}
In this note we will construct a simply-connected finite $\mathrm{CW}$ complex $X$ of dimension four with the following property. For each positive integer $m$, there is an element $\omega_{m}$ of order $m$ in $\pi_{m+1}(\Omega X)$ whose Hurewicz image $h\left(\omega_{m}\right) \in H_{m+1}(\Omega X)$ also has order $m$. Such a space illustrates how intricate loop space homology can be even for relatively simple spaces.
\end{abstract}

Our approach is first to construct a certain non-commutative graded Hopf algebra over $\mathbf{Z}$, which we call $A$. Next we will realize $A$ topologically by a simply-connected four-dimensional space $X$ in the sense that $A$ will be the image in $H_{*}(\Omega X)$ of $H_{*}\left(\Omega X^{2}\right)$, where $X^{2}$ is the 2-skeleton of $X$. The desired properties of $X$ will then follow from the properties of $A$. We will use the very simple commuting diagram

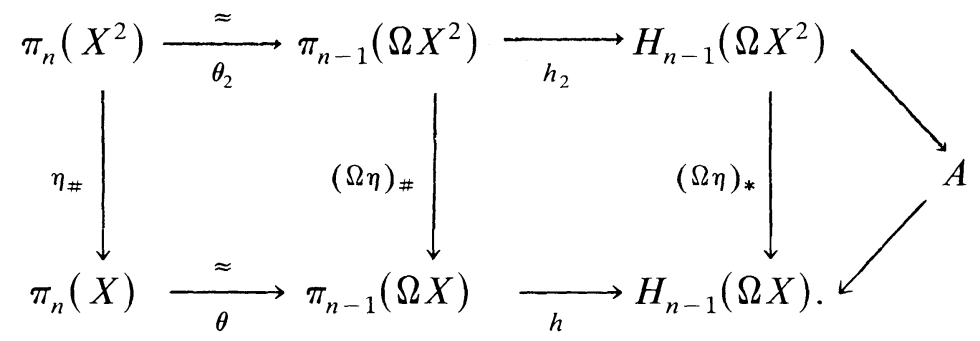

Here $\eta: X^{2} \rightarrow X$ is the inclusion; $h$ and $h_{2}$ denote Hurewicz homomorphisms; and $\theta$ and $\theta_{2}$ are the familiar isomorphisms.

For any ring $R$, let $R\left\langle y_{1}, \ldots, y_{n}\right\rangle$ denote the free associative algebra over $R$ with (non-commuting) generators $y_{1}, \ldots, y_{n}$. The $R$-algebra $R\left\langle y_{1}, \ldots, y_{n}\right\rangle$ becomes a (non-negatively) graded ring by allowing each $y_{l}$ to have degree one and assigning all of $R$ to degree zero. If $T$ is a graded ring ("graded" here will always mean graded by the non-negative integers), write $|x|$ for the degree of a homogeneous non-zero element $x$. The commutator of $x$ and $y$ in $T$ is

$$
[x, y]=x y-(-1)^{|x| \cdot|y|}(y x) \text {. }
$$

The Hopf algebra $A$ we seek will be constructed as the semi-tensor product [8] or smash product [4] of the free Hopf algebra $\mathbf{Z}\left\langle x_{1}, x_{2}\right\rangle$ with 
another algebra we call $E$. The ring $E$ is a quotient of the free $\mathbf{Z}$-algebra on five generators.

Put $F=\mathbf{Z}\left\langle u_{1}, u_{2}, u_{3}, v, w\right\rangle$. In $F$, for $i=1,2,3$ and for $m \geq 1$ recursively define $\sigma_{i, 1}=u_{i}$ and $\sigma_{i, m+1}=\left[\sigma_{i, m}, v\right]$. Then set

$$
\rho_{l, m}=\left[\sigma_{l, m-1}, w\right]=\left[\left[\ldots[u_{\imath}, \underbrace{v], \ldots, v]}_{(m-2) v ' s}, w]\right.\right.
$$

for $m \geq 2$. Lastly, let $\tau_{m}=\rho_{1, m}+(m) \rho_{2, m}$ for $m \geq 2$.

Put $E=F / I$, where $I$ is the two-sided ideal in $F$ generated by the set

$$
S=\left\{\tau_{m}\right\}_{m \geq 2} \cup\left\{(m) \rho_{3, m+1}\right\}_{m \geq 2} .
$$

Allow the graded Hopf algebra $D=\mathbf{Z}\left\langle x_{1}, x_{2}\right\rangle$ to act on the free algebra $F$ as follows:

$$
\begin{array}{ll}
x_{1} * u_{1}=\sigma_{1,2}+\sigma_{2,2} & x_{2} * u_{1}=0 \\
x_{1} * u_{2}=\sigma_{2,2} & x_{2} * u_{2}=\sigma_{3,2} \\
x_{1} * u_{3}=x_{1} * v=x_{1} * w=0 & x_{2} * u_{3}=x_{2} * v=x_{2} * w=0
\end{array}
$$

The reader is welcome to check that

$$
\begin{array}{ll}
x_{1} * \tau_{m}=\tau_{m+1} & x_{2} * \tau_{m}=(m) \rho_{3, m+1} \\
x_{1} * \rho_{3, m+1}=0 & x_{2} * \rho_{3, m+1}=0 .
\end{array}
$$

Consequently $D=\mathbf{Z}\left\langle x_{1}, x_{2}\right\rangle$ also acts on the quotient ring $E$. We can therefore define the semi-tensor product [8] of $D$ with $E$ :

$$
A=D \odot E \text {. }
$$

The algebra $A$ has a presentation as

$$
A=\mathbf{Z}\left\langle x_{1}, x_{2}, u_{1}, u_{2}, u_{3}, v, w\right\rangle / J,
$$

where $J$ is the two-sided ideal generated by the following eleven quadratic relations:

$$
\begin{array}{ll}
r_{1}=\left[x_{1}, u_{1}\right]-\left[u_{1}, v\right]-\left[u_{2}, v\right] & r_{7}=\left[x_{2}, u_{2}\right]-\left[u_{3}, v\right] \\
r_{2}=\left[x_{1}, u_{2}\right]-\left[u_{2}, v\right] & r_{8}=\left[x_{2}, u_{3}\right] \\
r_{3}=\left[x_{1}, u_{3}\right] & r_{9}=\left[x_{2}, v\right] \\
r_{4}=\left[x_{1}, v\right] & r_{10}=\left[x_{2}, w\right] \\
r_{5}=\left[x_{1}, w\right] & r_{11}=\left[u_{1}, w\right]+2 \cdot\left[u_{2}, w\right] . \\
r_{6}=\left[x_{2}, u_{1}\right] &
\end{array}
$$

The first ten of these relations describe the action of $D$ on $F$. The eleventh relation then has as consequences all the elements of the set (3); this is verified by the formulas (4). 
The algebra $A$ is made a Hopf algebra in a natural way by specifying that each of the seven generators be primitive and that the coproduct be cocommutative. We observe next that $A$ then has primitive torsion elements of any order. The obvious candidate to be a torsion element of order $m$ is $\rho_{3, m+1}$ (rigorously speaking, the image of $\rho_{3, m+1}$ under the obvious map $F \rightarrow E \hookrightarrow A)$. We must convince ourselves that $b \cdot \rho_{3, m+1} \neq$ 0 in $A$ unless $b \equiv 0(\bmod m)$. Because the semi-tensor product is isomorphic as a graded group with the tensor product, this is equivalent to the assertion that $b \cdot \rho_{3, m+1} \notin I \subset F$ unless $m \mid b$. However, this latter assertion is nearly obvious when one defines an additional grading on $F$ by setting $e\left(u_{1}\right)=e\left(u_{2}\right)=1$ but $e\left(u_{3}\right)=e(v)=e(w)=0$. Then $b \cdot \rho_{3, m+1}$ lies in the e-degree zero component of $F$ but it is not in the ideal generated by $\left(\rho_{3, j}\right)_{j \leq m}$ (except when $b=0$ ), so it belongs to $I$ only when $m \mid b$. Also note the obvious fact that $\rho_{3, m+1}$ is not itself a proper scalar multiple of any other element of $A$.

REMARK. The fact that $A$ has primitive torsion elements of any order is really its key property for the purposes of this note. Interestingly, it is not the first known example of a finitely presented graded $\mathbf{Z}$-algebra satisfying this condition. In [5, end of §3] a Hopf algebra denoted $C$ is built which also has primitive torsion elements of any prime order. Presumably it would not be too hard to verify that $C$ has torsion elements of any order, although that kind of information is not nearly so accessible for $C$ as it is for $A$. Also, the presentation for $C$ has fifteen generators and sixty-nine relations, considerably more than the seven-eleven combination (5). For these reasons and in order to offer a more self-contained exposition, the author chose to give the explicit description of the algebra $A$.

The discussion now moves into algebraic topology. We can neatly relate the Whitehead product in $\pi_{*}(X)$ and the Pontrjagin multiplication in $H_{*}(\Omega X)$ via Samelson's formula [7],

$$
h \theta([\beta, \gamma])=-(-1)^{\operatorname{deg}(\beta)}[h \theta(\beta), h \theta(\gamma)],
$$

the bracket on the left denoting Whitehead product and the bracket on the right denoting commutator. Knowing this, it makes sense to interpret the brackets in (5) as Whitehead products. Define the CW complex $X$ to be

$$
X=\bigvee_{i=1}^{7} S^{2} \cup\left(\bigcup_{j=1}^{11} e^{4}\right)
$$

where the attaching maps for the eleven 4-cells are described via (5). The Adams-Hilton construction assures us that $H_{*}(\Omega X) \approx H_{*}(B, d)$, 
where $(B, d)$ is the free differential graded algebra with $B=$ $\mathbf{Z}\left\langle x_{1}, x_{2}, u_{1}, u_{2}, u_{3}, v, w, e_{1}, \ldots, e_{11}\right\rangle$ and $d\left(e_{j}\right)=r_{j},\left|e_{j}\right|=3$. Clearly $A$ is precisely the subalgebra of $H_{*}(\Omega X)$ generated by $H_{1}(\Omega X)$, i.e., the subalgebra which equals $\operatorname{im}(\Omega \eta)_{*}$.

Since $A$ is a subalgebra of $H_{*}(\Omega X)$, the torsion elements we found in $A$ also lie in $H_{*}(\Omega X)$. Thus we have fulfilled the claim implicit in the title, i.e., $X$ is a finite simply-connected $\mathrm{CW}$ complex of dimension four such that $H_{*}(\Omega X)$ has torsion elements of every order.

We will take this example a little further and verify two more properties of $\Omega X$. First, the torsion elements we found will be shown to fall within the image of the Hurewicz homomorphism. Second, we will compute the Poincaré series of $\Omega X$ for each possible characteristic $p$.

The first claim is almost trivial. Let $\alpha_{m-1}$ be the repeated Whitehead product in $\pi_{m+1}\left(X^{2}\right)$ described via the right-hand side of (2) with $i=3$, and put $\omega_{m}=\theta \eta_{\#}\left(\alpha_{m}\right) \in \pi_{m+1}(\Omega X)$. By (6) we have

$$
h\left(\omega_{m}\right)= \pm \rho_{3, m+1} \text {, }
$$

as desired.

The interesting question here is this: does $h\left(m \cdot \omega_{m}\right)$ equal zero in $H_{m+1}(\Omega X)$ because $m \cdot \omega_{m}$ vanishes already in $\pi_{m+1}(\Omega X)$, or is $m \cdot \omega_{m}$ merely another non-zero element in the kernel of the Hurewicz map? In fact the former occurs. To see this, let $K$ be the smallest $\mathbf{Z}$-submodule of $H_{*}\left(\Omega X^{2}\right)$ which contains the $\left\{r_{j}\right\}$ and which is closed under taking commutators with elements of degree one. The module $K$ is smaller than the Lie ideal generated by the $\left\{r_{j}\right\}$ in that squares of odd-degree elements are not adjoined. Nevertheless one quickly checks that $\left[x_{1}, \tau_{m}\right]-\tau_{m+1} \in K$ and that $\left[x_{2}, \tau_{m}\right]-m \cdot \rho_{3, m+1} \in K$. Since $\tau_{2}=r_{11}$, each $\tau_{m}$ and consequently each $m \cdot \rho_{3, m+1}$ belongs to $K$. We can write

$$
m \cdot \rho_{3, m+1}=\sum\left(\left[\ldots\left[r_{J}, \ldots\right], \ldots\right]\right),
$$

the right-hand side representing a suitable element of $K$.

Again interpret the eleven relations (5) as Whitehead products lying in $\pi_{3}\left(X^{2}\right)$. Letting $M$ denote the smallest $\mathbf{Z}$-submodule of $\pi_{*}\left(X^{2}\right)$ which contains the $\left\{r_{j}\right\}$ and which is closed under taking Whitehead products with $\pi_{2}\left(X^{2}\right)$, formula (6) assures us that $h_{2} \theta_{2}(M)=K$. Since $\left.h_{2} \theta_{2}\right|_{M}$ is injective, we know that $\pm\left(m \cdot \alpha_{m}\right)$ equals the right-hand side of (7) when the brackets in (7) are reinterpreted as Whitehead products in $\pi_{*}\left(X^{2}\right)$. Because $\left\{r_{J}\right\} \subset \operatorname{ker}\left(\eta_{\#}\right)$ we have $m \cdot \alpha_{m} \in \operatorname{ker}\left(\eta_{\#}\right)$ and $m \cdot \omega_{m}=$ $\theta \eta_{\#}\left(m \cdot \alpha_{m}\right)=0$. Thus $\pi_{*}(\Omega X)$ has torsion elements of every order which are detected by the Hurewicz map. In particular, the homotopy of $X$ cannot have an exponent at any prime $p$. 
Let $\mathbf{Z}_{p}$ denote the prime field of characteristic $p$. Working over $\mathbf{Z}_{p}$, the Poincaré series of $\Omega X$ is the formal power series

$$
P_{\Omega X}(z)=\sum_{n=0}^{\infty} \operatorname{dim}\left(H_{n}\left(\Omega X ; \mathbf{Z}_{p}\right)\right) z^{n} .
$$

A well-known formula (e.g., [3, Theorem 3.7]) asserts that for our $X$ this is related to the Hilbert series of the graded $\mathbf{Z}_{p}$-algebra $A \otimes \mathbf{Z}_{p}$ by the formula

$$
P_{\Omega X}(z)^{-1}=(1+z)\left(A \otimes \mathbf{Z}_{p}\right)(z)^{-1}-z+7 z^{2}-11 z^{3} .
$$

To compute this for a given $p$, note that because $A$ is isomorphic as a graded abelian group with $D \otimes E$ we have

$$
\begin{aligned}
\left(A \otimes \mathbf{Z}_{p}\right)(z) & =\left(\mathbf{Z}\left\langle x_{1}, x_{2}\right\rangle \otimes \mathbf{Z}_{p}\right)(z) \cdot\left(E \otimes \mathbf{Z}_{p}\right)(z) \\
& =(1-2 z)^{-1} \cdot\left(E \otimes \mathbf{Z}_{p}\right)(z) .
\end{aligned}
$$

Fortunately, $E \otimes \mathbf{Z}_{p}$ is a graded algebra over a field whose ideal of relations is generated by (3), and for each $p$ the non-zero elements in (3) form a strongly free set [2]. We therefore know

$$
\begin{aligned}
&\left(E \otimes \mathbf{Z}_{p}\right)(z)^{-1}= 1-5 z+z^{2}(1+z)(1-z)^{-1} \\
&-z^{p}\left(1-z^{p}\right)^{-1} \quad \text { if } p \neq 0 ; \\
&\left(E \otimes \mathbf{Z}_{p}\right)(z)^{-1}=1-5 z+z^{2}(1+z)(1-z)^{-1} \quad \text { if } p=0 .
\end{aligned}
$$

Thus the Poincaré series of $\Omega X$ yields a different rational function for each possible characteristic $p$.

A final point of interest lies in the fact that the $m$-torsion we found for $H_{*}(\Omega X)$ occurred in $H_{m+1}(\Omega X)$. By increasing the number of cells and the complexity of the algebras involved one can obtain a space $Y$ for which $m$-torsion occurs in $H_{[m / a]+3}(\Omega Y)$, the bracket denoting the greatest integer function, for any positive integer $a \geq 2$. With still more cells (but sticking to 1-connected finite $\mathrm{CW}$ complexes of dimension four) there is a $W$ such that $m$-torsion occurs in $\pi_{\left[\log _{a} m\right]+5}(W)$ and $H_{\left[\log _{a} m\right]+4}(\Omega W)$.

This suggests a natural conjecture. Given a space $S$ and a prime $p$, let $t=t_{S}(p)$ denote the smallest integer (or if none exists let $t=\infty$ ) such that $\pi_{t}(S)$ has $p$-torsion. For $S$ a simply-connected finite $\mathrm{CW}$ complex, is it true that

$$
t_{S}(p) \geq O(\log p) ?
$$

Because each $\pi_{m}(S)$ is finitely generated we know that $t_{S}(p) \rightarrow \infty$ as $p \rightarrow \infty$. But how slowly can this sequence grow? 
Note. Luchezar Avramov has also constructed an example of a loop space whose homology has torsion of every prime order. His example will appear in Topology.

\section{REFERENCES}

[1] J. F. Adams and P. J. Hilton, On the chain algebra of a loop space, Comm. Math. Helv., 30 (1955), 305-330.

[2] D. Anick, Non-commutative graded algebras and their Hilbert series, J. Algebra, 78 No. 1 (1982), 120-140.

[3] _ A counterexample to a conjecture of Serre, Annals of Math., 115 (1982), 1-33.

[4] G. Bergman, Everybody knows what a Hopf algebra is, Contemporary Math., 43 (1985), 25-48.

[5] R. Fröbrg, T. Gulliksen and C. Löfwall, Flat Families of Local Artinian Algebras with an Infinite Number of Poincaré Series, Reports No. 4 (1984), Matematiska Institutionen, Stockholms Universitet, Stockholm, Sweden.

[6] I. M. James, Reduced product spaces, Annals of Math., 62 (1955), 170-197.

[7] H. Samelson, A Connection between the Whitehead and the Pontrjagin product, Amer. J. Math., 75 (1953), 744-752.

[8] L. Smith, Split extensions of Hopf algebras and semi-tensor products, Math. Scand., 26 (1970), 17-41.

Received February 26, 1985. Partially supported by NSF grant \# DMS-8303257.

MaSSACHUSETTS INSTITUTE OF TeCHNOLOGY

CAMBRIDGE, MA 02139 


\title{
PACIFIC JOURNAL OF MATHEMATICS EDITORS
}

\author{
V. S. VARADARAJAN \\ (Managing Editor) \\ University of California \\ Los Angeles, CA 90024 \\ HERBERT ClEMENS \\ University of Utah \\ Salt Lake City, UT 84112 \\ R. FINN \\ Stanford University \\ Stanford, CA 94305
}

\author{
HERMANN FLASCHKA \\ University of Arizona \\ Tucson, AZ 85721 \\ RAMESH A. GANGOLLI \\ University of Washington \\ Seattle, WA 98195 \\ VAUGHAN F. R. JONES \\ University of California \\ Berkeley, CA 94720 \\ ROBION KIRBY \\ University of California \\ Berkeley, CA 94720
}

C. C. MOORE

University of California

Berkeley, CA 94720

H. SAMELSON

Stanford University

Stanford, CA 94305

HAROLD STARK

University of California, San Diego

La Jolla, CA 92093

\section{ASSOCIATE EDITORS}

\author{
R. AREnS \\ E. F. BECKENBACH \\ B. H. NEUMANN \\ F. WOLF \\ K. YOSHIDA \\ (1906-1982)

\section{SUPPORTING INSTITUTIONS}

\begin{abstract}
UNIVERSITY OF ARIZONA
UNIVERSITY OF BRITISH COLUMBIA

UNIVERSITY OF CALIFORNIA

MONTANA STATE UNIVERSITY

UNIVERSITY OF NEVADA, RENO

NEW MEXICO STATE UNIVERSITY

OREGON STATE UNIVERSITY
\end{abstract} \\ CALIFORNIA INSTITUTE OF TECHNOLOGY \\ UNIVERSITY OF OREGON \\ UNIVERSITY OF SOUTHERN CALIFORNIA \\ STANFORD UNIVERSITY \\ UNIVERSITY OF HAWAII \\ UNIVERSITY OF TOKYO \\ UNIVERSITY OF UTAH \\ WASHINGTON STATE UNIVERSITY \\ UNIVERSITY OF WASHINGTON
}

The Supporting Institutions listed above contribute to the cost of publication of this Journal, but they are not owners or publishers and have no responsibility for its content or policies.

Mathematical papers intended for publication in the Pacific Journal of Mathematics should be in typed form or offset-reproduced (not dittoed), double spaced with large margins. Please do not use built up fractions in the text of the manuscript. However, you may use them in the displayed equations. Underline Greek letters in red, German in green, and script in blue. The first paragraph must be capable of being used separately as a synopsis of the entire paper. In particular it should contain no bibliographic references. Please propose a heading for the odd numbered pages of less than 35 characters. Manuscripts, in triplicate, may be sent to any one of the editors. Please classify according to the scheme of Math. Reviews, Index to Vol. 39. Supply name and address of author to whom proofs should be sent. All other communications should be addressed to the managing editor, or Elaine Barth, University of California, Los Angeles, California 90024.

There are page-charges associated with articles appearing in the Pacific Journal of Mathematics. These charges are expected to be paid by the author's University, Government Agency or Company. If the author or authors do not have access to such Institutional support these charges are waived. Single authors will receive 50 free reprints; joint authors will receive a total of 100 free reprints. Additional copies may be obtained at cost in multiples of 50 .

The Pacific Journal of Mathematics is issued monthly as of January 1966. Regular subscription rate: $\$ 190.00$ a year (5 Vols., 10 issues). Special rate: $\$ 95.00$ a year to individual members of supporting institutions.

Subscriptions, orders for numbers issued in the last three calendar years, and changes of address should be sent to Pacific Journal of Mathematics, P.O. Box 969, Carmel Valley, CA 93924, U.S.A. Old back numbers obtainable from Kraus Periodicals Co., Route 100, Millwood, NY 10546.

The Pacific Journal of Mathematics at P.O. Box 969, Carmel Valley, CA 93924 (ISSN 0030-8730) publishes 5 volumes per year. Application to mail at Second-class postage rates is pending at Carmel Valley, California, and additional mailing offices. Postmaster: send address changes to Pacific Journal of Mathematics, P.O. Box 969, Carmel Valley, CA 93924.

PUBLISHED BY PACIFIC JOURNAL OF MATHEMATICS, A NON-PROFIT CORPORATION

Copyright (C) 1986 by Pacific Journal of Mathematics 


\section{Pacific Journal of Mathematics}

\section{Vol. 123, No. $2 \quad$ April, 1986}

David Jay Anick, A loop space whose homology has torsion of all orders . . 257 Steven P. Diaz, Space curves that intersect often ................. 263

Thierry Fack and Hideki Kosaki, Generalized $s$-numbers of $\tau$-measurable

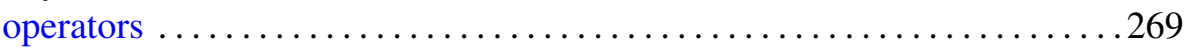

Karl Heinrich Hofmann and Karl Strambach, Lie's fundamental

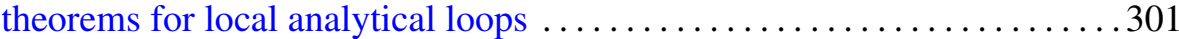

James Secord Howland, On the Kato-Rosenblum theorem ............. 329

Frieder Knüppel and Edzard Salow, Plane elliptic geometry over rings . . . . 337

Alan Noell, Peak points in boundaries not of finite type ................ 385

William J. Ralph, An extension of singular homology to Banach

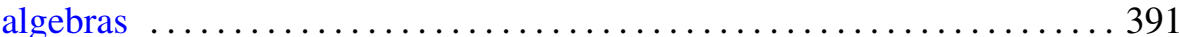

Wade C. Ramey, Averaging properties of pluriharmonic boundary values . . 407

Thomas Joseph Ransford, On the range of an analytic multivalued

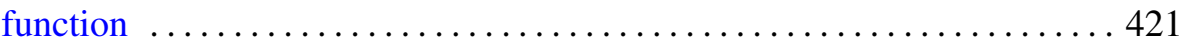

Christopher Donald Sogge, On restriction theorems of maximal-type . . . . 441

Edwin Spanier, Cohomology with supports $\ldots \ldots \ldots \ldots \ldots \ldots \ldots \ldots \ldots 47$

Emil J. Straube, Orthogonal projections onto subspaces of the harmonic

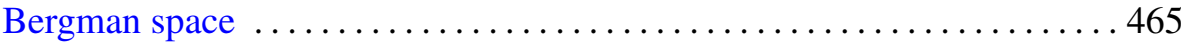

Thomas Vogel, Asymptotic behavior of two semilinear elliptic free boundary problems $\ldots \ldots \ldots \ldots \ldots \ldots \ldots \ldots \ldots$ 